\title{
Does Online Search Behavior Coincide with Candida auris Cases? An Exploratory Study
}

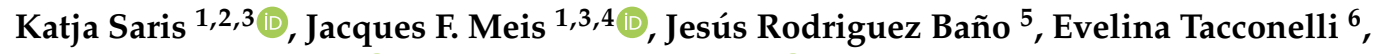 \\ Tom H. van de Belt ${ }^{2,7}$ (D) and Andreas Voss 1,2,3,*(D) \\ 1 Department of Medical Microbiology and Infectious Diseases, Canisius-Wilhelmina Hospital (CWZ), \\ 6532SZ Nijmegen, The Netherlands; katjasaris@gmail.com (K.S.); jacques.meis@gmail.com (J.F.M.) \\ 2 REshape Center for Innovation, Radboudumc, 6525GA Nijmegen, The Netherlands; \\ tom.vandebelt@radboudumc.nl \\ 3 Department of Medical Microbiology, Radboudumc, 6525GA Nijmegen, The Netherlands \\ 4 Center of Expertise in Mycology, Radboudumc/CWZ, 6525GA Nijmegen, The Netherlands \\ 5 Department of Medicine, Infectious Diseases, Microbiology and Preventive Medicine Unit, \\ Hospital Universitario Virgen Macarena, University of Sevilla, IBiS, 41009 Seville, Spain; jesusrb@us.es \\ 6 Department of Diagnostic and Public Health, Infectious Diseases, University of Verona, 37129 Verona, Italy; \\ evelina.tacconelli@univr.it \\ 7 Radboud Institute for Health sciences, Radboudumc, 6525GA Nijmegen, The Netherlands \\ * Correspondence: a.voss@cwz.nl
}

Received: 15 April 2019; Accepted: 19 May 2019; Published: 4 June 2019

check for updates

\begin{abstract}
Candida auris is an emerging multidrug resistant infectious yeast which is challenging to eradicate and despite available laboratory methods is still difficult to identify especially in less developed countries. To limit the rapid spread of C. auris, quick and accurate detection is essential. From the perspective of disease surveillance, additional methods of tracking this yeast are needed. In order to increase global preparedness, we explored the use of online search behavior to monitor the recent global spread of $C$. auris. We used Google Trends to assess online search behavior on C. auris from January 2016 until August 2018. Weekly Google Trends results were counted as hits and compared to confirmed C. auris cases obtained via publications and a global expert network of key opinion leaders. A total of 44 countries generated a hit, of which $30 \%(13 / 44)$ were confirmed known cases, 34\% (15/44) were missed known cases, 34\% (15/44) were hits for unknown cases, and $2 \%(1 / 44)$ were confirmed unknown cases. Conclusions: Google Trends searches is rapidly able to provide information on countries with an increased search interest in C. auris. However, Google Trends search results do not generally coincide with $C$. auris cases or clusters. This study did show that using Google Trends provides both insight into the known and highlights the unknown, providing potential for surveillance and tracking and hence aid in taking timely precautionary measures.
\end{abstract}

Keywords: Candida auris; cases; Google Trends; search behavior; surveillance

\section{Introduction}

Candida auris is an emerging multidrug resistant infectious yeast which is fast spreading globally and has reached all inhabited continents [1]. It predominantly spreads in the nosocomial setting. Since the first report of C. auris in 2009 [2], this yeast has caused increasing numbers of infections and outbreaks around the globe [3-9]. Candida auris causes bloodstream infections, wound infections, and otitis $[10,11]$. It mainly affects vulnerable patient groups such as intensive care patients and neonates treated in health care facilities [12]. Additionally, due to the predominant nosocomial spread of $C$. auris, it is important that health care facilities be aware and ready in case of C. auris infection (case) or outbreak (cluster) $[10,13]$. Outbreaks in Europe and the USA could be related 
to introductions of $C$. auris into the healthcare environments from one or more of the four known clades [14]. Due to increasing awareness of health care workers, government agencies, and researchers and better biochemical identification methods due to updates in lab system databases, $C$. auris becomes easier to identify [15]. However, this is still not the case in less developed countries. Additionally, the multidrug resistant nature of C. auris together with its high virulence [12], its capability to survive from weeks to months in the environment of hospital surfaces, and difficulty in decolonization $[7,16]$ are reasons for grave concern and raised global awareness and alarm [9,17-19]. This is so especially as we are still looking for the best way to track the spread of $C$. auris not just globally but also locally within institutions [20-23]. Therefore, there is a pressing need for accurate, timely, and efficient ways of tracking C. auris.

Since the Internet is widely used, it is an interesting source of information which is often used for academic research such as analysing (micro) blog content or global online search behavior [24]. In particular, the Internet has shown to be a valuable source to detect and monitor disease outbreaks such as the flu and others $[25,26]$. Online search behavior has also successfully been used to predict other infectious diseases including Methicillin-resistant Staphylococcus aureus (MRSA) outbreaks based on search entries [27]. Since $C$. auris cases are often location specific, online search behavior data could be a valuable source of information about emerging $C$. auris cases and clusters. We explored the use of online search behavior to monitor the recent global spread of $C$. auris in order to increase global preparedness.

\section{Materials and Methods}

\subsection{Design and Setting}

An exploratory global study was conducted in which online search behavior on Candida auris was compared to confirmed $C$. auris cases or clusters. The latter were derived from scientific peer reviewed publications of $C$. auris cases and clusters, searches in grey literature, and internationally conducted mini surveys within an expert network of key opinion leaders (KOLs) asking about knowledge of national $C$. auris cases or occurrence of clusters.

Online search behavior assessment on C. auris was performed between 1 January 2016 and 31 July 2018. Key opinion leaders were asked during 2017 and the first half of 2018 to use their network to provide unpublished information on cases or clusters in their country of residence. The KOLs in mycology and/or infection control were selected from those countries that showed activity in their online search behavior for $C$. auris but had no published occurrence of cases or clusters in the literature within the set search interval.

\subsection{Identifying C. auris Cases and Clusters}

A search for C. auris was conducted in the scientific databases PubMed and Web of Science for the years 2016 to 2018. Additionally, references in the literature were checked and, when relevant, added to the literature list. Grey literature was selected based on it being mentioned in the reference list of selected scientific publications. Websites of interest for grey literature were public health websites and conference websites in the field of microbiology and mycology. Literature had to be in English. Literature on scientific evaluations, such as antifungal resistance analyses or typing, and reports based only on previously obtained isolates without cases or clusters of $C$. auris detected between 2016 and the middle of 2018 were excluded.

Key opinion leaders in the field were identified and consulted via expert contacts in the Dutch field of Infection Control and Mycology. The latter sent messages to KOLs in 12 of 16 countries without publications but with Google Trends activity or a hit, (Table 1) asking about their knowledge on C. auris cases or clusters in their country and the month and year of the occurrence between 2016 and the middle of 2018. Additionally, the experts gathered information from KOLs at the 20th Congress of the International Society of Human and Animal Mycology (ISHAM 30 June until 4 July 2018 in The 
Netherlands). Replies of the KOLs provided data used for this study including any unpublished studies or data on C. auris. A report on C. auris was included if a KOL reported a cluster, infection, or potential index case within the country in the given timeframe.

Table 1. Overview of $C$. auris in published literature, Google Trends and key opinion leaders (KOL) from 2016 to the middle of 2018.

\begin{tabular}{ccc}
\hline $\begin{array}{c}\text { Lit+ GT+ } \\
(\mathbf{n}=\mathbf{1 3})\end{array}$ & $\begin{array}{c}\text { Lit+ GT- } \\
(\mathbf{n}=\mathbf{1 5})\end{array}$ & $\begin{array}{c}\text { GT+ Lit- } \\
(\mathbf{n}=\mathbf{1 6})\end{array}$ \\
\hline Austria & Bangladesh & Australia \\
\hline Belgium & China & Belarus * \\
\hline Canada & France & Brazil \\
\hline Colombia & Israel & Bulgaria \\
\hline Germany & Japan & Chile* \\
\hline India & Kuwait & Croatia \\
\hline Panama & Malaysia & Finland \\
\hline Spain & Norway & Ireland \\
\hline Switzerland & Oman & Italy \\
\hline UK & Pakistan & Mexico \\
\hline United Arab Emirates & Russia & The Netherlands \\
\hline USA & Saudi Arabia & Peru * \\
\hline Venezuela & Singapore & Philippines * \\
\hline & South Africa & Poland \\
\hline & South Korea & Sweden \\
\hline & & Thailand \\
\hline
\end{tabular}

Light grey shading is KOL positive $(n=1)$, dark grey shading is KOL negative $(n=15),{ }^{*}$ No KOL confirmation was obtained.

\subsection{Assessing Online Search Behavior}

To assess online search behavior, Google Trends (GT) was used [28]. This tool provides insight into search behavior of all people using Google based on specific searches performed in Google Search. It provides the relative frequency of searches for different countries and regions globally in the specified time interval. We used a similar approach as has been used in studies that successfully detected influenza and MRSA outbreaks using GT [26,27]. Although different search engines are used worldwide, our searches were limited to Google Trends since Google covers approximately $82 \%$ of all Internet searches [29].

Regarding the searches, only the term Candida auris was used since this term is indisputable, clear and comprehensive. No specific settings for GT were applied other than the search region that was set to worldwide. Google Trends presents search interest of topics on a scale ( $0 \%$ to $100 \%)$ per day instead of an absolute number thus every set search interval will have a maximum and a minimum even when absolute search numbers are low during this interval (e.g. without any peaks in search behavior) [27]. Since C. auris is relatively new, with the first case reported in 2009 [2], and outbreaks have not been reported in every country, search intervals were set to one week, starting on 1 January 2016 when C. auris picked up global interest. In addition, as of January 2016, Google Trends improved their system on information gathering [28]. The location (country) with the highest (100\%) search interest for the term Candida auris per week was gathered and defined as a hit. The hits were added to our database.

\subsection{Analysis}

A Table was created based on the finding of $C$. auris in the literature/KOL information and the GT hits, see Table 1. In addition, information from the literature/KOL and GT was combined and added to a table in alphabetical order (number of cases, country, and period, see Table S1). Countries with overlap in C. auris data collection and GT hit were added to a separate table if information on 
timeframe including month and year of cases was available. Detection of overlap in timeframe is interesting as potential for case or cluster identification.

\subsection{Ethical Approval}

Since the anonymous data used in this study was derived from the public social media domain without patient involvement and pre-existing contacts of specialists in the field were used, no medical ethical review was needed in The Netherlands.

\section{Results}

Between 1 January 2016 and August 2018, we performed searches in GT resulting in hits in 29 countries. Twelve KOLs were consulted, resulting in 1 unknown case in 1 country (Australia).

Table 1 shows the combination of the GT hits with published and unpublished C. auris reports within the search period. The first column (Lit+ GT+) shows the countries with published reports on C. auris cases or clusters between 2016 and the middle of 2018 with at least one hit in GT during the search period $(n=13)$. More countries had published reports on $C$. auris but showed no hits in the GT search $(n=15)$, which is shown in the second column (Lit+ GT-). The largest group had no published reports on C. auris cases or clusters but had at least one hit in the GT search (GT+ Lit-) $(n=16)$. Finally, the KOL information was used to assess whether countries with GT hits but no present literature were indeed without cases. Consequently, the final column shows the true positive countries (GT+, Lit-, $\mathrm{KOL}+)$ in light grey $(n=1)$ and false positive countries for $C$. auris cases or clusters (GT+, Lit-, KOL-) in dark grey $(n=15)$.

The combined literature search and GT hit collection, resulted in a total of 44 countries. Of these, $30 \%(13 / 44)$ were identified known C. auris cases by GT search, $34 \%(15 / 44)$ were missed known C. auris cases by GT search behavior, $2 \%(1 / 44)$ were confirmed unknown C. auris cases, and $34 \%(15 / 44)$ were confirmed hits for unknown cases.

Countries with overlap in C. auris data collection presented in the literature and GT hit within the set search interval were Colombia, the UK and the USA. All three publications provided a date range for case or cluster collection (see Table 2).

Table 2. A light grey shading of the overlap in the published C. auris cases or clusters and GT hits for 2016 to the middle of 2018.

\begin{tabular}{|c|c|c|}
\hline Country & Timeframe (and Number of Cases) & GT hits \\
\hline Colombia & February-July 2016 (17) [5] & $\begin{array}{c}\text { July 2016, August } 2016(2 \times) \text {, October } 2016 \\
(4 \times) \text {, February 2017, July 2017, August 2017, } \\
\text { December 2017, March 2018, April 2018, } \\
\text { May } 2018(2 \times)\end{array}$ \\
\hline $\mathrm{UK}^{\mathrm{a}}$ & $\begin{array}{l}2 \text { February 2015-31 August } 2017 \text { (70) [23] } \\
\text { April 2015-July } 2016 \text { (50) [7] }\end{array}$ & $\begin{array}{l}\text { January 2016, March } 2016(3 \times) \text {, April 2016, } \\
\text { May 2016, June 2016, August } 2016(2 \times) \text {, } \\
\text { December 2016, February } 2017(2 \times) \text {, August } \\
\text { 2017, March 2018, April } 2018\end{array}$ \\
\hline $\mathrm{USA}^{\mathrm{a}}$ & $\begin{array}{c}\text { May 2013-August } 2016 \text { (7) of which } 5 \text { in } \\
\text { April-August 2016 [30] }\end{array}$ & $\begin{array}{l}\text { June 2016, July 2016, March 2017, April } \\
\text { 2017, March } 2018 \text { (2×) }\end{array}$ \\
\hline
\end{tabular}

${ }^{a}$ On 1 July 2016, both the CDC and Public Health England (PHE) issued the first notification for C. auris.

\section{Discussion}

In this study, we explored the potential value of assessing online search behavior on Candida auris in relation to $C$. auris cases or clusters. One third of the hits found in GT were already known C. auris cases, well described in the literature. It is encouraging that a freely available, easy-to-access tool such as GT shows these cases. Additionally, our approach rapidly provided us with several new 
insights into $C$. auris cases, including the finding of one country which indeed had a C. auris case not known publicly. Interestingly, one third of the GT hits were not confirmed by official data from publications or KOLs. On the other hand, one third of known C. auris cases were not identified via online search behavior.

One third $(34 \%)$ of the hits were not confirmed by literature or KOLs. However, we cannot conclude that these hits are missed $C$. auris cases, particularly since not all hits were confirmed by KOL reply. This finding suggests that one third of the countries had an increased search interest for $C$. auris within the 1.5 year set-time interval as these countries peaked in GT. This increased interest could be on any subject regarding C. auris. However, for a country to come up as a hit in this study, it means that online search interest within this country was higher than in any other nation, suggesting an increased interest in C. auris and a potential increase in awareness which is essential for the adequate identification, treatment, and prevention of this yeast pathogen. This finding highlights the potential of using online search behavior such as via GT for governments and researchers as a new way of gathering information, which is strengthened by the knowledge that we live in a digital world in which we can assume that $C$. auris is discussed online.

We used Google Trends to identify C. auris cases as has been done for influenza and MRSA outbreaks previously. However, it is best used for high prevalence, non-emerging but prevalent diseases, which is not yet the case for C. auris [26]. Candida auris infections are relatively new and the identification, treatment, and prevention measures are not completely understood and applicable worldwide [9]. Therefore $C$. auris might not have the prevalence for proper cluster or case detection via Google Trends. Especially, since many published C. auris reports consist of $<10$ cases [6,30-32]. Additionally, the overlap found in published C. auris case collection and GT hit was minimal with only three countries out of the 28 countries reported to have C. auris cases or clusters (Tables 1 and 2). Moreover, even for these three countries, the hits in GT currently cannot be linked exclusively to the published case collection. However, it is to be expected that the 'critical mass' for C. auris detection via Internet will increase as this yeast continues to spread globally, infection cases become more common and well reported, and the potential for using social media and GT in particular becomes more valuable.

Even though the Internet is indeed worldwide, there is still a difference between more developed countries and developing countries in its use and accessibility [33]. This might have contributed to the low $(2 \%, n=1)$ number of developed countries (Australia) with an identified unknown case and the higher number of missed known cases (34\%) in countries on the African and Asian continents with potentially less internet access. However, in our current fast-changing digital era, it is essential that we explore new ways to track and detect diseases. Using the Internet is one of those methods [24].

Furthermore, as misidentification of $C$. auris is still a problem in many countries [12], C. auris might go undetected even when present. Since detection is a predicament in some facilities that are not using up-to-date equipment or standards [34], possible C. auris isolates might be sent to foreign laboratories for confirmation. Identification in a facility in another country can possibly result in a skewed peak search interest in the country of detection rather than the country of C. auris isolate origin. This might be true, for example, for cases detected in the USA that originated in the Middle East and infections detected in the UK that had an origin in South Africa $[9,23]$. This potentially contributes to the relatively high percentage of missed known cases (34\%) and identified hits for unknown cases (34\%) found. Additionally, search activity could have been influenced by publications from national organisations such as the CDC and PHE. However, this study does show that people are searching for C. auris and that this yeast is becoming more and more well-known globally.

\section{Limitations}

The timeframe for the publications to be included was set to either case collection or duration of the study between 2016 and the middle of 2018 or (first) publication from 2016 to the middle of 2018 . This timeframe was chosen to coincide with the Google Trends search period. It did include some of the recent major outbreaks, such as known reported outbreaks in the UK and South Africa [7,35]. 
However, to create Table 1, the timeframe used did not take into account differences in date of the GT hit and publication or KOL information date on C. auris within the set timeframe.

Since GT shows the relative frequency of searches for different countries and regions in a specified time interval, the result differs depending on the time interval chosen. However, since we used search intervals of a week, we believe this represents true search behavior better than looking at monthly GT searches.

Finally, we only looked at Candida auris as search term in Google Trends. This can result in a variety of hits on this topic. In this exploratory study we have specifically looked in the literature and networks for $C$. auris cases and clusters, whereas Google searches conducted with the term Candida auris may have been looking for information on resistance or detection of $C$. auris and therefore might give a skewed image for the number of local hits counted as cases or clusters. However, interest in C. auris in any form might be an indication of infection or potential cases and at least of increased awareness.

\section{Conclusions}

Google Trends searches rapidly provide information on countries with an increased search interest in C. auris. Currently, this freely available, easy-to-access tool is encouraging research by trying to correlate search behavior for $C$. auris in countries with known infections or cases. However, at the moment online search behavior for $C$. auris generates a relatively high misidentification of published cases and hits for unknown cases and shows little overlap in timeframe for case collection and Google Trends search behavior. Therefore, it can be concluded that Google Trends searches currently do not generally coincide with $C$. auris cases or clusters. More research is needed, especially at a time when C. auris becomes endemic and thus does not trigger searches by those trying to get informed about an emerging problem. At that point, Google Trends might be better suited to identify peaks in occurrence, e.g., outbreaks, as it does for influenza [25]. Particularly in a fast-changing digital world, health care organisations, professionals, and researchers are prompted to look for new methods of surveillance and earlier detection of diseases. This study shows that using such a tool both provides insight into the known and highlights the unknown, providing potential for surveillance and tracking and hence aid in taking timely precautionary measures.

Supplementary Materials: The following are available online at http://www.mdpi.com/2309-608X/5/2/44/s1, Table S1: Overview of (un)published C. auris outbreaks or cases, KOL information and GT hits 2016-first half 2018.

Author Contributions: K.S. and A.V. designed the study, after consulting with J.R.B. and E.T. K.S. collected and analyzed data and produced a draft of the manuscript, and T.H.v.d.B., J.F.M., J.R.B. and E.T. and A.V. reviewed it at various stages to its final version. All authors read and approved the final manuscript.

Funding: This research was supported by the EPI-Net COMBACTE-MAGNET project. We thank the Innovative Medicines Initiative Joint Undertaking for supporting the EPI-Net COMBACTE-MAGNET project (grant agreement number 115737), resources that include a financial contribution from the European Union Seventh Framework Programme (FP7/2007-2013) and a European Federation of Pharmaceutical Industries and Associations companies in-kind contribution. The funders had no role in study design, data collection and analysis, decision to publish, or preparation of the manuscript.

Conflicts of Interest: J.F.M. received grants from Astellas, Basilea, F2G, and Merck; J.F.M. has been a consultant to Astellas, Basilea, Scynexis, and Merck and has received speaker's fees from Astellas, Merck, United Medical, TEVA, and Gilead.

\section{References}

1. Saris, K.; Meis, J.F.; Voss, A. Candida auris. Curr. Opin. Infect. Dis. 2018, 31, 334-340. [CrossRef] [PubMed]

2. Satoh, K.; Makimura, K.; Hasumi, Y.; Nishiyama, Y.; Uchida, K.; Yamaguchi, H. Candida auris sp. nov., A novel ascomycetous yeast isolated from the external ear canal of an inpatient in a Japanese hospital. Microbiol. Immunol. 2009, 53, 41-44. [CrossRef] [PubMed]

3. Chowdhary, A.; Sharma, C.; Duggal, S.; Agarwal, K.; Prakash, A.; Singh, P.K.; Jain, S.; Kathuria, S.; Randhawa, H.S.; Hagen, F.; et al. New clonal strain of Candida auris, Delhi, India. Emerg. Infect. Dis. 2013, 19, 1670-1673. [CrossRef] [PubMed] 
4. Magobo, R.E.; Corcoran, C.; Seetharam, S.; Govender, N.P. Candida auris-associated candidemia, South Africa. Emerg. Infect. Dis. 2014, 20, 1250-1251. [CrossRef] [PubMed]

5. Morales-Lóópez, S.E.; Parra-Giraldo, C.M.; Ceballos-Garzón, A.; Martínez, H.P.; Rodríguez, G.J.; Álvarez-Moreno, C.A.; Rodríguez, J.Y. Invasive infections with multidrug-resistant yeast Candida auris, Colombia. Emerg. Infect. Dis. 2017, 23, 162-164. [CrossRef] [PubMed]

6. Ruiz Gaitan, A.C.; Moret, A.; Lopez Hontangas, J.L.; Molina, J.M.; Aleixandre Lopez, A.I.; Cabezas, A.H.; Mollar Maseres, J.; Arcas, R.C.; Gomez Ruiz, M.D.; Chiveli, M.A.; et al. Nosocomial fungemia by Candida auris: First four reported cases in continental Europe. Rev. Iberoam. Micol. 2017, 34, 23-27. [CrossRef] [PubMed]

7. Schelenz, S.; Hagen, F.; Rhodes, J.L.; Abdolrasouli, A.; Chowdhary, A.; Hall, A.; Ryan, L.; Shackleton, J.; Trimlett, R.; Meis, J.F.; et al. First hospital outbreak of the globally emerging Candida auris in a European hospital. Antimicrob. Resist. Infect. Control. 2016, 5, 35. [CrossRef] [PubMed]

8. Tsay, S.; Welsh, R.M.; Adams, E.H.; Chow, N.A.; Gade, L.; Berkow, E.L.; Poirot, E.; Lutterloh, E.; Quinn, M.; Chaturvedi, S.; et al. Notes from the field: Ongoing transmission of Candida auris in health care facilities United States, June 2016-May 2017. MMWR Morb. Mortal. Wkly. Rep. 2017, 66, 514-515. [CrossRef]

9. Centers for Disease Control and Prevention (CDC). Tracking C. auris. Available online: https://www.cdc.gov/ fungal/candida-auris/tracking-c-auris.html (accessed on 20 April 2018).

10. Lee, W.G.; Shin, J.H.; Uh, Y.; Kang, M.G.; Kim, S.H.; Park, K.H.; Jang, H.C. First three reported cases of nosocomial fungemia caused by Candida auris. J. Clin. Microbiol. 2011, 49, 3139-3142. [CrossRef]

11. Choi, H.I.; An, J.; Hwang, J.J.; Moon, S.Y.; Son, J.S. Otomastoiditis caused by Candida auris: Case report and literature review. Mycoses 2017, 60, 488-492. [CrossRef]

12. Jeffery-Smith, A.; Taori, S.K.; Schelenz, S.; Jeffery, K.; Johnson, E.M.; Borman, A.; Candida auris Incident Management, T.; Manuel, R.; Brown, C.S. Candida auris: A review of the literature. Clin. Microbiol. Rev. 2018, 31, e00029-00017. [CrossRef] [PubMed]

13. Chowdhary, A.; Voss, A.; Meis, J.F. Multidrug-resistant Candida auris: 'New kid on the block' in hospital-associated infections? J. Hosp. Infect. 2016, 94, 209-212. [CrossRef] [PubMed]

14. Lockhart, S.R.; Etienne, K.A.; Vallabhaneni, S.; Farooqi, J.; Chowdhary, A.; Govender, N.P.; Colombo, A.L.; Calvo, B.; Cuomo, C.A.; Desjardins, C.A.; et al. Simultaneous Emergence of Multidrug-Resistant Cida auris on 3 Continents Confirmed by Whole-Genome Sequencing and Epidemiological Analyses. Clin. Infect. Dis. 2017, 64, 134-140. [CrossRef] [PubMed]

15. Kordalewska, M.; Zhao, Y.; Lockhart, S.R.; Chowdhary, A.; Berrio, I.; Perlin, D.S. Rapid and accurate molecular identification of the emerging multidrug-resistant pathogen Candida auris. J. Clin. Microbiol. 2017, 55, 2445-2452. [CrossRef] [PubMed]

16. Welsh, R.M.; Bentz, M.L.; Shams, A.; Houston, H.; Lyons, A.; Rose, L.J.; Litvintseva, A.P. Survival, persistence, and isolation of the emerging multidrug-resistant pathogenic yeast Candida auris on a plastic health care surface. J. Clin. Microbiol. 2017, 55, 2996-3005. [CrossRef] [PubMed]

17. European Centre for Disease Prevention and Control (ECDC). Candida auris in Healthcare Settings-Europe; ECDC, Ed.; ECDC: Stockholm, Sweden, 2016.

18. Public Health England (PHE). Guidance for the Laboratory Investigation, Management and Infection Prevention and Control for Cases of Candida auris; The Department of Health, Ed.; Public Health England: London, England, 2016; p. 16.

19. Chowdhary, A.; Sharma, C.; Meis, J.F. Candida auris: A rapidly emerging cause of hospital-acquired multidrug-resistant fungal infections globally. PLoS Pathog. 2017, 13, e1006290. [CrossRef] [PubMed]

20. Ruiz-Gaitan, A.; Moret, A.M.; Tasias-Pitarch, M.; Aleixandre-Lopez, A.I.; Martinez-Morel, H.; Calabuig, E.; Salavert-Lleti, M.; Ramirez, P.; Lopez-Hontangas, J.L.; Hagen, F.; et al. An outbreak due to Candida auris with prolonged colonisation and candidaemia in a tertiary care European hospital. Mycoses 2018, 61, 498-505. [CrossRef]

21. Biswal, M.; Rudramurthy, S.M.; Jain, N.; Shamanth, A.S.; Sharma, D.; Jain, K.; Yaddanapudi, L.N.; Chakrabarti, A. Controlling a possible outbreak of Candida auris infection: Lessons learnt from multiple interventions. J. Hosp. Infect. 2017, 97, 363-370. [CrossRef] [PubMed] 
22. Chow, N.A.; Gade, L.; Tsay, S.V.; Forsberg, K.; Greenko, J.A.; Southwick, K.L.; Barrett, P.M.; Kerins, J.L.; Lockhart, S.R.; Chiller, T.M.; et al. Multiple introductions and subsequent transmission of multidrug-resistant Candida auris in the USA: A molecular epidemiological survey. Lancet Infect. Dis. 2018, 18, 1377-1384. [CrossRef]

23. Eyre, D.W.; Sheppard, A.E.; Madder, H.; Moir, I.; Moroney, R.; Quan, T.P.; Griffiths, D.; George, S.; Butcher, L.; Morgan, M.; et al. A Candida auris outbreak and its control in an intensive care setting. N. Engl. J. Med. 2018, 379, 1322-1331. [CrossRef]

24. Mavragani, A.; Ochoa, G.; Tsagarakis, K.P. Assessing the methods, tools, and statistical approaches in google trends research: Systematic review. J. Med. Internet. Res. 2018, 20, e270. [CrossRef] [PubMed]

25. Ortiz, J.R.; Zhou, H.; Shay, D.K.; Neuzil, K.M.; Fowlkes, A.L.; Goss, C.H. Monitoring influenza activity in the United States: A comparison of traditional surveillance systems with google flu trends. PLoS ONE 2011, 6, e18687. [CrossRef] [PubMed]

26. Carneiro, H.A.; Mylonakis, E. Google trends: A web-based tool for real-time surveillance of disease outbreaks. Clin. Infect. Dis. 2009, 49, 1557-1564. [CrossRef] [PubMed]

27. Van de Belt, T.H.; van Stockum, P.T.; Engelen, L.J.L.P.G.; Lancee, J.; Schrijver, R.; Rodríguez-Baño, J.; Tacconelli, E.; Saris, K.; van Gelder, M.M.H.J.; Voss, A. Social media posts and online search behaviour as early-warning system for MRSA outbreaks. Antimicrob Resist. Infect. Control. 2018, 7, 69. [CrossRef] [PubMed]

28. Google. Google Trends. Available online: https://trends.google.nl/trends/ (accessed on 1 April 2019).

29. Nationale Search Engine Monitor. (iprospect en rmi). Available online: https://www.iprospect.com/nl/nl/ press-room/nationale-search-engine-monitor-onderzoek/ (accessed on 1 April 2019).

30. Vallabhaneni, S.; Kallen, A.; Tsay, S.; Chow, N.; Welsh, R.; Kerins, J.; Kemble, S.K.; Pacilli, M.; Black, S.R.; Landon, E.; et al. Investigation of the first seven reported cases of Candida auris, a globally emerging invasive, multidrug-resistant fungus-United States, May 2013-August 2016. MMWR. Morb. Mortal. Wkly. Rep. 2017, 65, 1234-1237. [CrossRef] [PubMed]

31. Arauz, A.B.; Caceres, D.H.; Santiago, E.; Armstrong, P.; Arosemena, S.; Ramos, C.; Espinosa-Bode, A.; Borace, J.; Hayer, L.; Cedeno, I.; et al. Isolation of Candida auris from 9 patients in Central America: Importance of accurate diagnosis and susceptibility testing. Mycoses 2018, 61, 44-47. [CrossRef] [PubMed]

32. Alatoom, A.; Sartawi, M.; Lawlor, K.; AbdelWareth, L.; Thomsen, J.; Nusair, A.; Mirza, I. Persistent candidemia despite appropriate fungal therapy: First case of Candida auris from the United Arab Emirates. Int. J. Infect. Dis. 2018, 70, 36-37. [CrossRef]

33. United Nations Statistics Division. Internet Users per 100 Inhabitants. Available online: http://data.un.org/ Data.aspx?q=internet+use\&d=MDG\&f=seriesRowID\%3a605 (accessed on 1 April 2019).

34. European Centre for Disease Prevention and Control (ECDC). Candida auris in Healthcare Settings-Europe; ECDC, Ed.; ECDC: Stockholm, Sweden, 2018.

35. Govender, N.P.; Magobo, R.E.; Mpembe, R.; Mhlanga, M.; Matlapeng, P.; Corcoran, C.; Govind, C.; Lowman, W.; Senekal, M.; Thomas, J. Candida auris in South Africa, 2012-2016. Emerg. Infect. Dis. 2018, 24, 2036-2040. [CrossRef]

(C) 2019 by the authors. Licensee MDPI, Basel, Switzerland. This article is an open access article distributed under the terms and conditions of the Creative Commons Attribution (CC BY) license (http://creativecommons.org/licenses/by/4.0/). 